\title{
TikTok sa likod ng mga mag-aaral sa komersyo
}

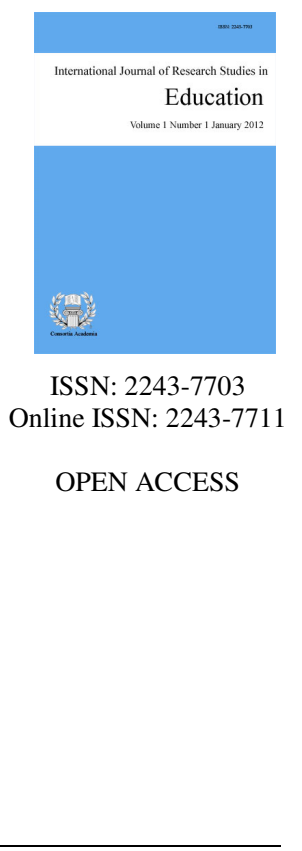

Kamantigue, Angelo $\measuredangle$

Veritas College of Irosin, Philippines (Angelo.kamantigue20@gmail.com)

Gueta, Maicky

Veritas College of Irosin, Philippines (ikaygueta@gmail.com)

Pura, Riena

Veritas College of Irosin, Philippines (purarienamae@gmail.com)

Enriola, Jaella

Veritas College of Irosin, Philippines (enriolajaella@gmail.com)

Gelilio, Eric $\triangle$

Veritas College of Irosin, Philippines (gelilioeric@ gmail.com)

Sorsogon State University, Philippines

Gallanosa National High School, Philippines

\section{Abstract}

One of the trends for students today is TikTok a video sharing social networking service that originated in China owned by Byte Dance. Used to create short videos of dance, lip-sync, comedy and talent, Tiktok is trending fast in social media, and we see this on Facebook, Instagram, and YouTube. The purpose of this study is to identify and evaluate the Tiktok behind the students in the first year of college in the commerce course at Veritas College of Irosin in the year 2021-2022. Descriptive analysis was the design used in the research. Random sampling was used to collect data from 100 student participants from the first year in the college of commerce. Ortiz (2015) also agrees that experimental research can actually test an assumption or hypothesis about the cause-and-effect relationship. This is often considered the most sophisticated technique of research to test the assumptions. Dilon's (2020) study revealed that with continued use of the TikTok application the users can become anti-social. Like other social applications, TikTok also does not require direct interaction with others for the formation of their own. It is said that young people spend their time using social media apps to spend time, have fun, and gather a variety of information. The study also shows that most users of applications like TikTok are shaping themselves and making it a way to overcome self-deficiencies through indirect interaction.

Keywords: Tiktok, variety of information, impacts, sophisticated techniques, self-deficiencies, indirect interaction 


\section{TikTok sa likod ng mga mag-aaral sa komersyo}

\section{Introduksyon}

Sa modernong henerasyon ngayon may mga makabagong teknolohiya na ang mga naimbento, ang komunikasyon ay naging mas madali sa lipunan lalo na sa paggamit ng social media. Ang isa sa mga pinaka-nakakaimpluwensyang social video app ay ang TikTok. Ang TikTok ay isang video sharing social networking service ng pagmamay-ari ng Byte Dance isang chinese company na itinatag noong 2012 ni Zhang Yiming. Ginagamit ito upang makapaglikha ng maikling bidyo ng sayaw, lip-sync, komedya, at talento, mabilis ang pag trending ng TikTok sa social media at ito ay nakikita natin sa Facebook, sa Instagram, at sa Youtube, isa ang TikTok sa mga naging libangan ng mga mga karamihang mag-aaral simula ng tayo ay napasa ilalim sa Lockdown dahil sa Pandemya. Kama-kailan napa-balita ang posibling pag ban ng TikTok sa ilang mga bansa kasama na ang Pilipinas dahil sa pagnanakaw nito ng pribadong impormasyon at ibinibigay sa gobyerno nga china. Ang TikTok ay napunta sa isang serye ng kontrobersya at noong 2019 ito ay pinagbawalan ng india sa maikling panahon dahil sa isyu sa moralidad at ilang pagkakataon na inakusahan ito ng hindi sapat na ginagawa upang protektahan ang mga gumagamit na sa pangkalahatan ay ang mga kabataang teenager at young adult. Inimbestigahan ang TikTok sa US dahil sa mga dalahanin sa pambansang siguridad ngunit sa kabila ng mga pagkukulang na ito, napatunayan ng TikTok na paglaban sa pamumuna at ano mang backlash mula sa mga kritiko.

Ayon sa ulat ni Kapuso broadcast Journalist Oscar Oida para sa 24 oras, wala na raw nakikitang dahilan ang malacañang para i-ban ang TikTok sa bansa. "wala pong kahit anong website ng na bina-ban ang Presidente. Wala pong dahilan na nakikita para i-ban ang TikTok dito sa Pilipinas," pahayag ni Presidential Spokesperson Harry Roque. Ayon kay Pace (2019), hindi na bago ang labis na paggamit ng batang henerasyon sa social media applications tulad ng Instagram o Twitter ngunit, ang TikTok application ay mabilis na pumatok sa masa sa maikling panahon. Bago maging TikTok ang pangalan ng aplikasyong ito, tinatawag itong Musical.ly at naging layunin ng bagong aplikasyon na ibahin ang imahe ng lumang programa para sa mga nakababatang gumagamit nito. Ayon din kay Dolye (2020), ang TikTok application ay magagamit sa mahigit kumulang 150 bansa at mayroong higit 1 bilyong kataong gumagamit ng naturang application na kung saan 850 milyong tao ang tinatayang aktibong gumagamit nito. Naging patok ito lalong-lalo na sa mga mag-aaral at halos $90 \%$ sa mga kabataan ang nahuhumaling at gumagamit ng TikTok sa kanilang pang araw-araw na pamumuhay. Ayon kay John Herman The Newyork Times (2019), babaguhin ng TikTok ang paraan ng paggana ng iyong social media kahit na iniiwasan mo ito. Bagama't maaaring tila hindi ito totoo mula sa unang impresyon, ang TikTok ay isang mahusay na video platform para sa edukasyon. Maraming online influencers ngayon ang gumagamit ng TikTok upang ibahagi ang edukasyon, negosyo, at career lesson.

Ang TikTok ay naging isang mahusay na mapagkukunan sa pag-aaral para sa lahat, kabilang ang mga kapaki-pakinabang na lifehacks, natatanging mga eksperimento sa agham, dapat matuto ng mga trick sa matematika, mabilis at madaling mga proyekto sa DIY, at mga pampasiglang mensahe at payo. Dahil ang app ay napakadaling gamitin, nahanap ng mga tagalikha na ito ay isang maginhawang lugar upang magbahagi ng kaalaman at kasanayan sa isang malawak na madla. Sa pamamagitan ng platform na ito, ang mga tagapagturo ay nakakita ng isang paraan upang buhayin ang mga aralin, na ginagawang mas nakakaengganyo, mabilis, at madaling matunaw. Kasabay nito, ang format ng maikling form na video ng TikTok ay gumagawa ng pag-akit ng micro learning sa mga gumagamit sa lahat ng edad. Sa TikTok hindi lamang mga video ng mga paboritong tanyang na tao ang iyong mapapanuod, kung uunawain mo ng maigi ang TikTok makikita mo ang mahusay na mga ideya ng TikTok upang makatulong saiyo bilang mag-aaral. TikTok ay isang mahusay na kasangkapan para sa edukasyon, gayunman, kapag ginamit sa panahon ng pag-aaral, ito ay maaaring maging isang malaking distraksyon sa pag-aaral. Ang pananaliksik na ito ay naglalayon sa mga kabataang mag-aaral ng mga Veritans sa 
unang taon ng kolehiyo sa Kursong Komersyo na sa dumaraming bilang na gumagamit ng TikTok at layunin ng pananaliksik na ito na matukoy ang impak ng TikTok at ang mga salik na nakakaapekto sa paglaganap nito sa mga mag-aaral sa komersyo.

\subsection{Paglalahad ng Suliranin}

Ang pananaliksik na ito ay nakatuon sa pangunahing layunin nito na kung saan ay matukoy ang TikTok sa likod ng mga mag-aaral sa unang taon sa kurso ng komersyo. Nilalayon nitong sagutin nang tiyak ang mga sumusunod na suliranin:

$>\quad$ Ano-ano ang impak ng TikTok sa mga mag-aaral sa komersyo?

$>\quad$ Ano ang mga salik na nakakaapekto sa paglaganap ng TikTok sa mga mag-aaral sa komersyo?

$>\quad$ Ano ang mga nabuong rekomendasyon batay sa resulta ng pag-aaral?

\section{Pamamaraang ginamit}

Ang naisagawang pananaliksik ay ginamitan ng deskriptiv-analisis upang matukoy ang TikTok sa likod ng mga mag-aaral sa komersyo. Random sampling ang ginamit ng mga mananaliksik sa 100 na napiling kalahok mula sa pangkat ng mga mag-aaral sa unang taon ng kolehiyo sa kursong komersyo sa Veritas College of Irosin, Sorsogon. Isinagawa ang pagsisisyasat sa pamamagitan ng online na survey sa pagkolekta ng mga datos upang mas mapadali ang pakikipag-ugnayan ng mga mananaliksik sa mga maag-aaral na kalahok sa pananaliksik na hindi na kailangan ng harapang panayam upang masunod ang pangkalusugang protokol at maiwasan ang posibling pagkalat ng sakit dahil sa kinakaharap na pandemya. Ang mga mananaliksik ay gumamit ng tseklist na talatanungan sa pangangalap ng datos. Ang mga mananaliksik ay lumikha ng sariling gawang online na talatanungan na magsisilbing instrumento sa pananaliksik. Google Survey Form at Tseklist ang pangunahing gamit upang makakalap ng sagot mula sa napiling mga kalahok. Ang disenyong paglalarawan o deskriptibo ay ang nakita ng mananaliksik na magiging mabisa sa pag-aaral na ito upang mas makakalap ng impormasyon na magigimg epektibo sa pananaliksik. Ayon kay Toquero (2013) ang Descriptive Survey method ay ang mga kaganapan sa pag-aaral na ginagamitan ng paglalarawang pananaliksik ay kinapapalooban ng pagtatala, paglalarawan, pagpapakahulugan, pagsusuri at paghahambing. Layunin ng ganitong disenyo na sistematikong mailarawan ang sitwasyon at kundisyon nang makatotohanan at buong katiyakan. Ito ay binubuo ng obserbasyon, pagsasagawa ng sarbey, panayam, standardized tests at case studies. Naniniwala ang mga mananaliksik na angkop ang disenyong ito para sa paksang ito sapagkat mas mapapadali ang pangangalap ng datos mula sa maraming repondente.

\section{Mga natuklasan}

\subsection{Negatibo at Positibong Impakng TikTok sa mga Mag-aaral ng unang taon ng kolehiyo}

\section{Talahanayan 1}

Negatibong Impak ng TikTok sa mga Mag-aaral ng Komersyo.

\begin{tabular}{lccccc}
\hline \multicolumn{1}{c}{ Negatibong Impak } & Block E & Block F & Block G & Frequency & Rango \\
\hline $\begin{array}{l}\text { Nawawalan ng pokus sa pag-aaral dahil sa } \\
\text { pagsubaybay sa mga video sa TikTok }\end{array}$ & 20 & 34 & 26 & 89 & 1 \\
$\begin{array}{l}\text { Adiksyon } \\
\begin{array}{l}\text { Problemang pag-kalusugan. Napapabayaan } \\
\text { ang sarili }\end{array}\end{array} \quad 14$ & 30 & 17 & 61 & 4 \\
Maaaring pag mulan ng Cyber bullying & 14 & 24 & 19 & 57 & 6 \\
\hline
\end{tabular}


Kamantigue, A., Gueta, M., Pura, R., Enriola, J., \& Gelilio, E.

Pagpapatuloy...

Pag-aaksaya ng oras

Pag-kalat ng mga maling impormasyon at

prebadong impormasyon

Contains Inappropriate Contents

Naisasawalang bahala ang mga

mahahalagang gawain

Nagiging dahilan ng pagbaba ng grado at

lebel ng performans bilang mag-aaral

Hindi nagagawa ang mga gawain sa

paaralan tulad ng Modyul at iba pang

aktibidad

$\begin{array}{lcccc}16 & 32 & 23 & 71 & 3 \\ 10 & 24 & 15 & 49 & 10 \\ & & & & \\ 14 & 20 & 19 & 53 & 8 \\ 11 & 28 & 20 & 59 & 5 \\ 15 & 21 & 20 & 56 & 7 \\ 15 & 36 & 28 & 79 & 2\end{array}$

Batay sa resulta sa talahanayan sa itaas, napag-alaman na ang pangunahing naging negatibong impak ng TikTok sa mga mag-aaral sa unang taon ng kolehiyo sa kursong Komersyo sa Veritas Colloge of Irosin ay nawawalan ng pokus sa pag-aaral dahil sa labis na pagsubaybay sa mga video sa TikTok na 89 ng mga kalahok ang sumagot mula sa 100 na mga respondante. Sinundan ng Hindi nagagawa ang mga gawain sa paaralan tulad ng Modyul at iba pang aktibidad na 79 ng mga kalahok ang sumagot. Ayon kina Calimlim, Dayro, at Mancilla (2016), maraming estudyante ay may nasabi sa hindi mabuting epekto ng social media gaya ng TikTok na nakadudulot ng pagbaba ng grado sa kanilang pag-aaral, kakulangan sa oras para sa pamilya at maaring mapalapit sa masamang gawain ng mga estudyante. Marami din nagsasabi na marami din naitutulong ang social media lalo na sa pakikipag-komunikasyon dahil napadadali nito ang pag-uusap ng dalawang tao na magkalayotulad ng mga ofw na nakakausap ang kanilang pamilya gamit ang social media.

Ayon din kay Pascual (2019), may dalawang bilyong tao ang aktibong gumagamit ng social media sa buong mundo at ito ay nakaaapekto sa kanilang buhay at edukasyon. Sinabi rin Asidao (2017), na ayon sa mga eksperto, nakaka-apekto ang madalas na internet access sa kalusugan ng ating utak. Nagdudulot ito ng "sleep deprivation" o dahilan upang hinditayo makatulog sa gabi. Sa kabilang banda, nagiging dahilan din ito sa pagkakaroon natin ngmaikling pasensya o pokus sa isang bagay. Napag-alaman na napakalaki ng impak ng TikTok sa mga mag-aaral ng komersyo sa negatibong paraan.

Talahanayan 2

Positibong Impak ng TikTok sa mga Mag-aaral ng Komersyo

\begin{tabular}{|c|c|c|c|c|c|}
\hline Positibong Impak & Block E & Block F & Block G & Frequency & Rango \\
\hline $\begin{array}{l}\text { Nakakakuha ng mga ideya patungkol sa mga } \\
\text { pag-aaral sa Komersyo }\end{array}$ & 17 & 33 & 27 & 77 & 1 \\
\hline Nakakatulong para ilibang ang sarili & 13 & 32 & 16 & 61 & 5 \\
\hline $\begin{array}{l}\text { Naibabahagi ang talento sa pamamagitan ng } \\
\text { TikTok }\end{array}$ & 18 & 30 & 19 & 67 & 4 \\
\hline Learning new thing/Gain of Knowlegde & 10 & 21 & 18 & 49 & 10 \\
\hline $\begin{array}{l}\text { Malayang nailalabas ng mga mag-aaral ang } \\
\text { kanilang mga saloobin }\end{array}$ & 14 & 23 & 23 & 56 & 7 \\
\hline $\begin{array}{l}\text { Natututo ng mga DIY (Do it Yourself) at } \\
\text { Lifehacks na maaaring makatulong sa } \\
\text { pang-araw-araw na gawain }\end{array}$ & 14 & 27 & 19 & 60 & 6 \\
\hline $\begin{array}{l}\text { Nagkakaroon ng kaalaman sa kasalukuyang } \\
\text { mga pangyayari }\end{array}$ & 17 & 32 & 26 & 74 & 2 \\
\hline $\begin{array}{l}\text { Napaglalapit ang mga gumagamit nito kahit } \\
\text { nasa malayong lugar }\end{array}$ & 13 & 21 & 18 & 52 & 8 \\
\hline $\begin{array}{l}\text { Naisusulong ang mga produktong nais } \\
\text { ibenta }\end{array}$ & 15 & 31 & 25 & 71 & 3 \\
\hline Napapadali ang paraan ng komunikasyon & 9 & 27 & 15 & 51 & 9 \\
\hline
\end{tabular}

Lumabas sa naging resulta na ang pangunahing positibong impak ng TikTok sa mga mag-aaral ng unang taon ng kolehiyo sa kursong komersyo ay Nakakakuha ng mga ideya patungkol sa mga pag-aaral sa Komersyo na 77 ng mga kalahok mula sa 100 na respondante ang sumagot.Ang paggamit ng mga maiikling bidyo ay isa sa mga pamamaraan na maiiugnay sa pagbabagong teknolohikal sa larangan ng edukasyon ngayon pagpapakita ng 
TikTok sa likod ng mga mag-aaral sa komersyo

mga business tricks at mga marketing strategy sa TikTok ay malaking tulong sa mga mag-aaral ng Komersyo. Nagiging daan ito upang mapanatili ang interes ng mag-aaral sa aralin, napauunlad ang kakayahan sa pag-iisa at maaari ring maging batayan sa hinaharap. Kaugnay nito, sang-ayon sa artikulo ni Escober (2020) na ang aplikasyong TikTok ay nagiging daan ng mga kabataan upang matugunan ang simpleng realidad ng buhay at pagbuo ng mga makatotohanang kontent. Ang aplikasyong ito ay umusbong noong taong 2016, at naglalaman ng iba't ibang bidyo na patuloy na tinatangkilik nang karamihan. Sinundan ng Nagkakaroon ng kaalaman sa mga kasalukuyang pangyayari na may bilang ng 74 na mga kalahok ang sumagot mula sa 100 na respondante.

Ayon sa pagbabalita ng ABS-CBN news caster na si Jacque Manabat sa TV Patrol, pang-aliw ang kadalasang hatid ng TikTok. Pero ngayon ginagamit na rin ito at iba pang social media platforms para magbahagi ng balita at impormasyon sa sari-saring kaalaman at isyu ng bayan, kabilang ang balitang pandemya at nalalapit na halalan. Kama-kailan napapanuod narin sa TikTok ang iksplosibong pagbabalita ng ABS-CBN at 24 Oras na tumatagal ng isang oras. Ini-rerekomenda ng mga mananaliksik na mas mabuting suriin ang mga balita o chismis na napapanuod sa TikTok upang maiwasan at hindi mabiktima ng Fake news.

\section{Talahanayan 3}

Ang mga Salik na nakakaapekto sa paglaganap ng TkTok sa mga mag-aaral sa Komersyo.

\begin{tabular}{|c|c|c|c|c|c|}
\hline $\begin{array}{l}\text { Mga salik na nakakaapekto sa paglaganap ng } \\
\text { TikTok sa mga mag-aaral sa Komersyo } \\
\text { (BSBA) }\end{array}$ & Block E & Block F & Block G & Frequency & Rango \\
\hline $\begin{array}{l}\text { Pagdami ng tumatangkilik at naiingganyo na } \\
\text { mag-download ng TikTok }\end{array}$ & 14 & 29 & 25 & 68 & 3 \\
\hline $\begin{array}{l}\text { Dahil sa impluwesya ng mga Social Media } \\
\text { TikTok Influencers }\end{array}$ & 17 & 25 & 21 & 63 & 6 \\
\hline $\begin{array}{l}\text { Pag-uso ng mga TikTok trends at nakikisali } \\
\text { sa mga Hashtag Challenge }\end{array}$ & 15 & 24 & 23 & 62 & 7 \\
\hline $\begin{array}{l}\text { Mga nauusong sayaw na maraming } \\
\text { gumagawa ng sarili nilang bersyon }\end{array}$ & 13 & 24 & 23 & 60 & 8 \\
\hline Patok na TikTok filters at video effects & 17 & 26 & 26 & 69 & 2 \\
\hline $\begin{array}{l}\text { Malalaswang content creator na nagpapakita } \\
\text { ng parte ng katawan }\end{array}$ & 11 & 22 & 24 & 57 & 9 \\
\hline $\begin{array}{l}\text { Mga bagong produkto na nais ibenta sa } \\
\text { TikTok: Live Selling }\end{array}$ & 15 & 23 & 26 & 64 & 5 \\
\hline $\begin{array}{l}\text { Nakakakuha ng mga ideya sa pagluluto: } \\
\text { Cooking Tutorial }\end{array}$ & 16 & 17 & 21 & 54 & 10 \\
\hline $\begin{array}{l}\text { Gustong magbahagi ng talento at magbigay } \\
\text { inspiration }\end{array}$ & 14 & 25 & 31 & 70 & 1 \\
\hline $\begin{array}{l}\text { Para maka-iwas sa sakit na Anxiety at } \\
\text { Depression dulot ng pandemya }\end{array}$ & 15 & 28 & 22 & 65 & 4 \\
\hline
\end{tabular}

Batay sa result ng talahanayan sa itaas, ang salik na nakakaapekto sa paglaganap ng TikTok ay ang mga kabataan o mag-aaral na nahihikayat na ibahagi ang kanilang talento at magbigay inspirasyon sa iba. Nakakatulong ang TikTok sa pagbuo kumpiyansa ng isang taong gumagamit nito at nagbibigay din ito ng aliw sa mga tao at nakakatulong ito para mas maipakita ang talento ng isang mag-aaral sa kung ano mang larangan.

Ang TikTok ay isang app na nagbibigay ng interface sa gumagamit nito gumawa ng mga maikling video at ipahayag ang kanilang pagiging natatangi at kasabay ng pagbibigay sa kanila ng pribilehiyong maging ang mga nagpapakalat at tumatangkilik ng kanilang mga nilikha. Ang TikTok ay ang app na nagbago sa tradisyonal na mode ng paggawa ng kulturang sikat. Ginawa ng social media ang pag-access ng madali ang impormasyon at komunikasyon sa buong mundo ay naging walang hangganan. Ang kulturang sikat ay nagkaroon ng mas malaking epekto dahil sa pandaigdigang senaryo ng komunikasyon at impormasyon. Ang Tik Tok ay ang perpektong halimbawa ng sikat kultura kung saan ipinapahayag ng mga tao ang kanilang sarili sa pamamagitan ng paggawa lip sync sa kanilang mga paboritong himig at sa babahagi ng talento sa pamamagitan ng TikTok at minamahal ang application na ito, ang mga tao ay may mataas na antas ng kasiyahan sa app. (Burton 2012) (Naisbitt, Naisbitt \&Philips 2001) (Sorells,2015). Pangalawa sa mga salik sa paglaganap ng TikTok ay ang patok na mga filters at videos effect na tinangkilik ng marami lalo na ng mga mag-aaral sa unang taon ng kolehiyo sa 
Kamantigue, A., Gueta, M., Pura, R., Enriola, J., \& Gelilio, E.

kursong komersyo.

Ayon kay Dolye (2020) Naging patok ito lalong-lalo na sa mga mag-aaral at halos $90 \%$ sa mga kabataan ang nahuhumaling dahil sa nakakalibang na mga filters na maaaring magamit habang nagbi-bidyo at dahil dito mas madalas ang paggamit nila Tiktok sa kanilang pang-araw-araw na pamumuhay.

\section{Kongkulsyon}

Batay sa natuklasan, nabuo ang sumusunod na kongklusyon:

$>\quad$ Basi sa negatibong impak ng TikTok sa mga mag-aaral ng unang taon ng kolehiyo sa kursong komersyo ng Veritas College of Irosin ay nagkaroon ng impak sa kanilang pag-aaral dahil sa sobrang pagsubaybay sa mga video sa TikTok ay nawawala sila sa pokus sa sobrang pagkalibang sa nasabing app. Maaari itong maging dahilan ng pagbaba ng kanilang grado at pagkakulang ng oras sa pamilya at problemang pangkalusugan.

$>\quad$ Ang TikTok ay isang malawak na social networking service at dahil dito maaari kang makakuha ng ideya patungkol sa pag-aaral sa kursong komersyo tulad ng Business tricks at Marketing strategy na malaking tulong sa mga mag-aaral sa komersyo na magagamit balang araw isa lamang ito sa mga positibong impak ng TikTok sa mga mag-aaral sa unang taong ng kolehiyo sa kursong komersyo.

$>\quad$ Pagbabahagi ng talento at pagbibigay inspirasyon ang nakitang dahilan sa paglaganap ng TikTok. Dahil sa pagsikat ng TikTok maraming mga mag-aaral ang sumubok at nagsimulang mag pamalas ng kanilang mga talento sa iba't ibang larangan at pag post ng mga inspiring videos na maaaring maging inspirasyon ng mga kapwa mag-aaral at mga kabataan.

> Nagkaroon ng positibong impak ang TikTok sa mga mag-aaral ng unang taon ng kolehiyo sa komersyo, maraming pag-aaral ang maibabahagi ng TikTok na maaaring magamit, binibigyan ng TikTok sa pagkakataon ang isang mag-aaral na magbahagi ng kanilang kaalaman at sa kabilang banda ay matututo mula sa ibang mga user. Malaya din nilang nailalabas ang kanilang mga saloobin at talento hindi lang sa pagsayaw at pagkanta kundi pati narin sa kani-kanilang mga katalinohan.

\subsection{Rekomendasyon}

Sa kabuuan, mas marami ang naging positibong impak ng TikTok sa mga mag-aaral sa unang taon sa kolehiyo sa kursong Komersyo ng Veritas College of Irosin. Batay sa resulta at kongklusyon ng pag-aaral na ito, ang sumusunod na mga rekomendasyon ay nabuo:

$>\quad$ Mas makakabuting ibalanse ang oras upang hindi mawalan ng pokus sa pag-aaral na maaaring maging dahilan ng pagbaba ng grado at lebel ng performans mo bilang mag-aaral. Ugaliing unahin ang mga importanteng gawain gaya ng Modyul at iba pag mga aktibidad sa paaaralan.

$>\quad$ Maaaring magamit ang TikTok bilang isang instrumento ng pagkatuto na makakatulong saiyong pag-aaral sa kolehiyo sa kursong komersyo. Mga business ideas at mga DIY (Do It Yourself) na proyekto at Lifehacks na maaari mong magamit bilang mag-aaral ng komersyo, sa sitwasyon natin ngayon sa pandemya mas mabuting ituon natin ang ating atensyon sa pag-aaral at kinakailangan ng gabay mula sa ating mga guro sa birtwal na pamamaraan.

$>\quad$ Magpatupad ng pagpupulong upang mabantayan at magabayan ang mga mag-aaral ng kanilang mga guro at magulang sa tamang paggamit ng mga social media apps kagaya ng TikTok. May mga masasamang video na maaaring mag impluwensya sa mga mag-aaral at mag-iwan ng kakaibang imahe sa kanilang kaisipan. Makakatulong ang pag kakaroon ng konsultasyon mula sa mga eksperto upang maiwasan ang adiksyon at mga negatibong epekto ng TikTok sa mga mag-aaral.

50 Consortia Academia Publishing (A partner of Network of Professional Researchers and Educators) 
TikTok sa likod ng mga mag-aaral sa komersyo

$>\quad$ Ang TikTok ay isang makabagong paraan ng pagkatuto maaari mong gamitin ang TikTok para sa pansariling aktibidad sa paaralan at maging instrumento upang mapalawak ang kaisipan. Maaari din gamitin ng mga guro ang TikTok bilang makabagong pandulog at estratehiya sa pagtuturo sa mga mag-aaral. Gamitin ang TikTok sa kapaki-pakinabang na paraan at wag sa pagbabahagi ng mga hindi makabuluhang bagay.

\section{Talasanggunian}

Asidao. (2017) Academia.edu.

https://www.academia.edu/42206677/Epekto_ng_Labis_na_Paggamit_ng_Social_Media_sa_mga_Aspe to_ng_Buhay_ng_mga_Mag_aaral?auto=download

Burton. (2012). Tik Tok the New Alternative Media for Youngsters for Online Sharing of Talent: An Analytical Study.

Calimlim, Dayro, at Mancilla. (2016) Epekto ng Labis na Paggamit ng Social Media sa mga Aspeto ng Buhay ng mga Magaaral.

https://www.academia.edu/42206677/Epekto_ng_Labis_na_Paggamit_ng_Social_Media_sa_mga_Aspe to_ng_Buhay_ng_mga_Mag_aaral?auto=download

Dolye. (2020) [Couse Hero] Sta. Teresa College, Implikasyon ng Tiktok Application sa Pag-aaral, Pagkatuto at Pag-uugali ng mga mag-aaral. https://www.coursehero.com/file/75384184/REVISED-Kabanata-1-3docx/

Escober. (2020). Tiktok: Bilang Makabagong Pandulog at Estratehiya Sa Pagtuturo. https://www.researchgate.net/publication/353204456_TIKTOK_BILANG_MAKABAGONG_PANDU LOG_AT_ESTRATEHIYA_SA_PAGTUTURO

Herman, J. (2019) The New York Times, How TikTok Is Rewriting the World. https://www.nytimes.com/2019/03/10/style/what-is-tik-tok.html

Jacque Manabat. TV Patrol, Biyernes, 9 Oktubre 2021. ABS-CBN News\#Halalan2022: TikTokers inilarawan ang kanilang 'ideal leader'

Oscar Oida. [7 August 2020] Kapuso Broadcast Journalist, 24 Oras, TikTok iba-ban sa https://Pilipinas.gmanetwork.com/entertainment/showbiznews/hobbies/67148/tiktok

Pace. (2019) [Course Hero] https://Teknolohiya-Ang-teknolohiya-ay-ang-teoretikal-na-kaalaman-ukol-sa-industriya-at/

Toquero. (2013). [coursehero.com/file]. https://www.nytimes.com/2019/03/10/style/what-is-tik-tok.html 
Kamantigue, A., Gueta, M., Pura, R., Enriola, J., \& Gelilio, E.

52 Consortia Academia Publishing (A partner of Network of Professional Researchers and Educators) 